Dhaka Univ. J. Sci. 60(1): 47-51, 2012 (January)

\title{
Determinants and Status of Vaccination in Bangladesh
}

Nasrin Afzal and Begum Zainab

Institute of Statistical Research and Training (ISRT), University of Dhaka, Dhaka-1000, Bangladesh

Received on 27.02.2011. Accepted for Publication on 17. 09. 2011

\begin{abstract}
The identification of the characteristics that have an influence on the vaccination coverage of children and the determination of the pattern of such influence are very important since the government can reschedule the policy to immunize each and every child. This paper examines the factors that manipulate the vaccination coverage in terms of five major vaccines using the Bangladesh Demographic and Health Survey (BDHS)-2007 data. The results strongly suggest that mother's education and economic status play a vital role significantly in improving the vaccination coverage. Besides, Khulna and Rajshahi have higher whereas Sylhet and Chittagong have lower immunization coverage than Dhaka. In addition, mother's exposure to media (newspaper, TV or radio) also improves the status of coverage both in the rural and urban areas in Bangladesh.
\end{abstract}

Key words: Negative Binomial Regression Model, Poisson regression model, Vaccination.

\section{Introduction}

Universal immunization of children under one year of age against the six major vaccine preventable diseases (tuberculosis, diphtheria, pertussis, tetanus, poliomyelitis, and measles) is one of the most cost-effective programs to reduce infant and child morbidity. The Expanded Program on Immunization (EPI) is a priority program for the government of Bangladesh. It follows the international guidelines recommended by the World Health Organization (WHO). According to the guidelines, children are considered fully immunized when they have received one dose of the vaccine against tuberculosis (BCG), three doses each of the vaccine against diphtheria, pertussis and tetanus (DPT), three doses of polio vaccine (excluding polio vaccine given at birth), and one dose of measles vaccine. Summing up all these doses we get a total of 11 doses. WHO recommends giving children all of these vaccines before their first birthday.

The BDHS-2007 collected data on childhood vaccinations for all surviving children born during the five-year period before the survey. We are interested in identifying factors that create a difference in taking all the recommended vaccines. This information can significantly contribute to the improvement of the immunization status of children in Bangladesh. Therefore, the issue of recognizing influential covariates has received considerable attention.

Only a few works were done so far in Bangladesh regarding the vaccination program. Quaiyum et al. (1997) have conducted a research on the impact of National Immunization Days (NIDs) on polio-related knowledge and practice of urban women in Bangladesh. In the study they analyzed the reasons behind the success of the first two NIDs held in Bangladesh in 1995 (March 16 and April 16) as part of that country's goal to eradicate poliomyelitis by the turn of the century. Their paper assesses the impact of these two polio NIDs in terms of the immunization coverage and change in knowledge about the disease among women living in Dhaka city, the capital of the country. Chowdhury et al (2003) examined inequalities in the use of, and access to, vaccination service in Bangladesh by analyzing national and small area-based datasets. The analysis showed that female children had lower immunization coverage than male children. Children residing in urban areas were more likely to be fully immunized than their rural counterparts.

This study has important applications from several aspects. Firstly, we have used BDHS-2007 data; the most recent national level survey done with USAID support. So, we can understand the current status of vaccination from this study. Secondly, we have used Poisson and Negative Binomial Regression models to analyze the data; a completely new approach to analyze vaccination status of the children in Bangladesh. And lastly, we have not only analyzed the vaccination status in Bangladesh but also compared it between rural and urban areas, which facilitates to identify the background characteristics that make a difference on the immunization coverage between these two areas.

The paper is organized as follows: Section two discusses data and empirical methodology, while Section three presents empirical results. In Section four concluding remarks are provided.

\section{Data and Methodology}

This study is conducted using Bangladesh Demographic and Health Survey (BDHS)-2007 data, the fifth BDHS undertaken in Bangladesh. A two-stage sampling technique was conducted for this survey. Information on vaccination coverage focuses on all living children aged under five years 
before the survey. According to the BDHS-2007 data, the number of living children aged five years or less was 5729 , out of which 3741 were from rural and 1988 were from urban area respectively. As influential characteristics for taking all the recommended vaccines, the considered variables are: sex of the child (SEX), birth order number (BORD), division of residence (DIVISION), mother's education (MEDU), economic status of the family (WEALTH), frequency of reading newspaper (PAPER), listening radio (RADIO) and watching TV (TV) of the mother.

Cameron et al (1998) first introduced Poisson regression as the standard method to model the count response data. However, the Poisson distribution assumes the equality of its mean and variance - a property that is rarely found in real data. Data that have greater variance than the mean are termed as over dispersed. Hilbe (2007) used Negative Binomial regression to model over dispersed Poisson data. When the Negative Binomial is used to model over dispersed Poisson count data, the distribution can be thought of as an extension to the Poisson model.

The foregoing brief discussion suggests some essential steps in our analysis. The first step is to observe the mean and variance of the response variable (i.e. frequency of taking all vaccines). If the mean and variance of the response variable are approximately equal we can use Poisson regression model. The Poisson regression model is given by:

$\log [E(Y)]=a+b_{1} X_{1}+b_{2} X_{2}+\ldots+b_{p} X_{p}$

where, $Y$ is the response variable and $X_{1}, X_{2}, \ldots, X_{p}$ are the covariates, $a$ is the constant and $b_{1}, b_{2}, \ldots, b_{p}$ are the coefficients of the covariates. Next, we will employ two tests of over dispersion where the Null Hypothesis $\left(H_{0}\right)$ is: mean and variance of the response variable are equal against the Alternative Hypothesis $\left(H_{0}\right)$ : variance exceeds the mean.

Deviance and Pearson Chi-Square statistics divided by their corresponding degrees of freedom are used for this purpose. The Deviance Statistics is defined as:

$$
G^{2}=2 \sum_{i=1}^{I} \sum_{j=1}^{J} O_{i j} \log \left(\frac{O_{i j}}{E_{i j}}\right)
$$

where, $O_{i j}=n_{i j}$ is the observed count and $E_{i j}$ is the expected count in cell $(i, j)$ under $H_{0}$. The Pearson Chi-square statistics is defined as,

$$
X^{2}=\sum_{i=1}^{I} \sum_{j=1}^{J}\left(\frac{O_{i j}-E_{i j}}{E_{i j}}\right)^{2}
$$

The expected count, $E_{i j}$ for equation (2) and (3) is calculated as:

$$
E_{i j}=\frac{\text { totol of } i^{\text {th }} \text { row } \times \text { totol of } j^{\text {th }} \text { colomn }}{\text { total of all observation }}
$$

Both the test statistics (Deviance and Pearson Chi-square) divided by their corresponding degrees of freedom, if greater than one indicates over-dispersion, i.e. the variance is greater than the mean.

Now, if overdispersion is present in the data, Negative Binomial Regression model, an extension of Poisson Regression model is used:

$$
\operatorname{var}(Y)=\exp \left(a+b_{1} X_{1}+b_{2} X_{2}+\ldots+b_{p} X_{p}\right)
$$

The parameters of equation (1) and (4) are estimated using maximum likelihood method. The log-likelihood functions of equation (1) and (4) are given by,

$$
l(\beta \mid X, Y)=\sum_{i=1}^{m}\left(y_{i}\left(\beta^{\prime} x_{i}\right)-e^{\beta^{\prime} x_{i}}\right)
$$

and

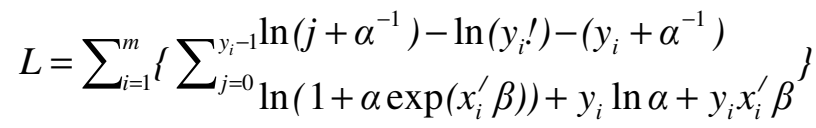

where equation (5) and (6) are log likelihood functions of Poisson and Negative Binomial regression models respectively, $m$ is the number of observation, $\beta$ is the parameter to be estimated and $\alpha$ is the dispersion parameter. Iterative techniques are applied in obtaining the maximum likelihood estimator.

\section{Results and Discussion}

Table 1 shows the rate of vaccination coverage among under five children by different selected characteristics. Results indicate that children in urban areas are more likely than children in rural area to be fully vaccinated. Girls and boys are almost equally likely to receive the basic immunizations in both the areas. Vaccination rate is highest for first child (36.7 percent in rural area and 42.8 percent in urban area).Among divisions; the highest level of coverage is seen in Khulna (38.8 percent) and the Rajshahi (49.1 percent) in rural and urban areas respectively. Mother's education and wealth are positively associated with children's likelihood of being fully vaccinated in both the areas. Similarly, mothers who read newspaper, listen to radio and watch TV almost every day fully vaccinate their children in both the areas than mothers who are not exposed to these media's every day. 
Table. 1. Percentage Distribution of children fully vaccinated by different characteristics in rural and urban area

\begin{tabular}{|c|c|c|c|}
\hline & & \multicolumn{2}{|c|}{ Percentage of Children taken all the vaccines } \\
\hline & & rural & Urban \\
\hline \multirow[t]{2}{*}{ SEX } & Female & $32.4 \%$ & $39.5 \%$ \\
\hline & Male & $32.3 \%$ & $41.3 \%$ \\
\hline \multirow[t]{4}{*}{ BORD } & 1st child & $36.7 \%$ & $42.8 \%$ \\
\hline & 2nd and 3rd child & $32.5 \%$ & $41.8 \%$ \\
\hline & 4th and 5th child & $29.9 \%$ & $31.1 \%$ \\
\hline & 6th child and above & $21.0 \%$ & $36.5 \%$ \\
\hline \multirow[t]{6}{*}{ DIVISION } & Dhaka & $32.3 \%$ & $39.1 \%$ \\
\hline & Barisal & $32.9 \%$ & $37.1 \%$ \\
\hline & Chittagong & $30.4 \%$ & $41.2 \%$ \\
\hline & Khulna & $38.8 \%$ & $45.7 \%$ \\
\hline & Rajshahi & $37.6 \%$ & $49.1 \%$ \\
\hline & Sylhet & $25.7 \%$ & $31.1 \%$ \\
\hline \multirow[t]{4}{*}{ MEDU } & No education & $25.5 \%$ & $28.4 \%$ \\
\hline & Primary & $29.7 \%$ & $35.8 \%$ \\
\hline & Secondary & $40.0 \%$ & $45.2 \%$ \\
\hline & Higher & $40.3 \%$ & $55.7 \%$ \\
\hline \multirow[t]{3}{*}{ WEALTH } & Poor & $28.6 \%$ & $29.1 \%$ \\
\hline & Middle Class & $31.5 \%$ & $33.3 \%$ \\
\hline & Rich & $40.4 \%$ & $44.8 \%$ \\
\hline \multirow[t]{4}{*}{ NEWSPAPER } & Not at all & $31.5 \%$ & $37.1 \%$ \\
\hline & Less than once a week & $36.1 \%$ & $51.9 \%$ \\
\hline & At least once a week & $40.9 \%$ & $45.7 \%$ \\
\hline & Almost everyday & $58.6 \%$ & $63.3 \%$ \\
\hline \multirow[t]{4}{*}{ RADIO } & Not at all & $31.7 \%$ & $40.1 \%$ \\
\hline & Less than once a week & $30.3 \%$ & $43.9 \%$ \\
\hline & At least once a week & $36.7 \%$ & $39.6 \%$ \\
\hline & Almost everyday & $34.1 \%$ & $43.1 \%$ \\
\hline \multirow[t]{4}{*}{ TV } & Not at all & $30.3 \%$ & $29.8 \%$ \\
\hline & Almost everyday & $32.1 \%$ & $37.6 \%$ \\
\hline & At least once a week & $32.9 \%$ & $43.1 \%$ \\
\hline & Less than once a week & $38.2 \%$ & $45.3 \%$ \\
\hline
\end{tabular}

We next extend our concentration to see the contribution of different covariates to vaccination coverage. To do so, first we need to determine the appropriate regression models for this data.

Table 2 represents the necessary statistics for selecting the appropriate regression model. Since the response variable (frequency of taking all recommended vaccines) is a count data and the mean and variance is just about equal we use the Poisson regression model.

The tests of overdispersion (Deviance Statistics and Pearson Chi-Square Statistics divided by their corresponding degrees of freedom) are greater than one; indicating over dispersion is present in the data and thus suggesting Negative Binomial
(NB) regression model except in one case. The exception is for urban area where the Pearson Chi-Square statistics divided by the d.f is almost one. To crack the ambiguity,

Table. 2. Summary Statistics and Tests of Overdispersion

\begin{tabular}{|l|cc|}
\hline Statistics & Rural & Urban \\
\hline Mean & 8.18 & 8.58 \\
Variance & 9.60 & 8.89 \\
\hline & \multicolumn{2}{|c|}{$\frac{\text { value }}{\text { d.f }}$} \\
\hline Deviance & $\frac{6372.94}{3716}=1.72$ & $\frac{2932.55}{1961}=1.50$ \\
\hline Pearson & $\frac{4281.33}{3716}=1.15$ & $\frac{1987.85}{1961}=1.03$ \\
Chi-Square & & \\
\hline
\end{tabular}


we will also use the Negative Binomial Regression model for urban area.

Table 3 represents the results obtained from fitting the regression models. Here $\log \mathrm{L}$ is the $\log$ likelihood of the regression models and $\mathrm{AIC}$ and $\mathrm{BIC}$ are the information criteria's for selecting the appropriate model. The lower the value of AIC and BIC, the better the model fits the data. For rural area both the AIC and BIC are smaller for Negative Binomial (NB) Regression model than Poisson Regression model indicating NB better fits the data. The opposite is true for urban area. Therefore, we will only make our remarks

Table. 3. Influence of different characteristics on vaccination coverage in rural and urban area

\begin{tabular}{|c|c|c|c|c|c|c|c|}
\hline & & & Rural & & & Urban & \\
\hline & & Poisson & NB & $\exp (\beta)$ & Poisson & $\exp (\beta)$ & NB \\
\hline $\log \mathrm{L}$ & & -10135.60 & -10104.91 & & -5242.89 & & -5242.88 \\
\hline AIC & & 20319.20 & 20287.24 & & 10533.77 & & 10535.77 \\
\hline BIC & & 20468.65 & 20442.91 & & 10668.01 & & 10675.60 \\
\hline SEX & Male & .0161 & .0181 & 1.02 & $.02861 * *$ & 1.03 & $.02865^{* *}$ \\
\hline BORD & $2^{\text {nd }}$ or $3^{\text {rd }}$ child & -.0165 & -.0174 & 0.98 & .01565 & 1.02 & .01565 \\
\hline & $4^{\text {th }}$ or $5^{\text {th }}$ child & -.0226 & -.0252 & 0.98 & -.02712 & 0.97 & -.02714 \\
\hline & $\geq 6^{\text {th }}$ child & $-.0864 * * *$ & $-.0916 * * *$ & 0.91 & $-.09248 * *$ & 0.91 & $-.09268 * *$ \\
\hline DIV & Barisal & .0098 & .0118 & 1.01 & -.00277 & 1.00 & -.00277 \\
\hline & Sylhet & $-.0684 * * *$ & $-.0741 * * *$ & 0.93 & -.03021 & 0.97 & -.03025 \\
\hline & Khulna & .0506 & $.0551 * *$ & 1.06 & .00154 & 1.00 & .00153 \\
\hline & Rajshahi & $.0390 * *$ & $.0409^{* *}$ & 1.04 & .01376 & 1.01 & .01374 \\
\hline & Chittagong & $-.1080 * * *$ & $-.1171 * * *$ & 0.89 & $-.12288 * * *$ & 0.88 & $-.12300 * * *$ \\
\hline MEDU & Primary & $.0463 * * *$ & $.0503 * * *$ & 1.05 & $.05455 * *$ & 1.06 & $.05461 * *$ \\
\hline & Secondary & $.1014 * * *$ & $.1067 * * *$ & 1.11 & $.09554 * * *$ & 1.10 & $.09562 * * *$ \\
\hline & Higher & $.0754 * *$ & $.0790 * *$ & 1.08 & $.13449 * * *$ & 1.14 & $.13459 * * *$ \\
\hline WEALTH & Middle Class & .0098 & .0100 & 1.01 & .04598 & 1.05 & .04604 \\
\hline & Rich & $.0415 * *$ & $.0417 * *$ & 1.04 & $.05107 * *$ & 1.05 & $.05111 * *$ \\
\hline PAPER & Sonce a week & .0302 & .0337 & 1.03 & .03087 & 1.03 & .03089 \\
\hline & At least once a week & .0338 & .0372 & 1.04 & -.01095 & 0.99 & -.01099 \\
\hline & Almost everyday & $.1295 * *$ & $.1343 * *$ & 1.14 & $.05953 *$ & 1.06 & $.05956^{*}$ \\
\hline RADIO & Sonce a week & -.0045 & -.0036 & 1.00 & .02688 & 1.03 & .02691 \\
\hline & At least once a week & -.0158 & -.0183 & 0.98 & -.00113 & 1.00 & -.00115 \\
\hline & Almost everyday & -.0128 & -.0145 & 0.99 & .00658 & 1.00 & .00658 \\
\hline TV & Sonce a week & -.0009 & .0013 & 1.00 & -.00374 & 1.00 & -.00378 \\
\hline & At least once a week & .0171 & .0206 & 1.02 & $.06446^{* * *}$ & 1.07 & $.0644794 * * *$ \\
\hline & Almost everyday & .0154 & .0187 & 1.02 & .03606 & 1.04 & .03608 \\
\hline CONST & & $2.0563 * * *$ & $2.0531 * * *$ & 7.79 & $2.00631 * * *$ & 7.44 & $2.00622 * * *$ \\
\hline Alpha & & & .2609 & & & & .00274 \\
\hline
\end{tabular}

NOTE: $* * * * *$ and $*$ denote that $\mathrm{p}$-value is less than $.01, .05$ and .10 respectively.

on this two best fitted regression models for the corresponding area. The best fitted models suggest that, the expected frequency of vaccination coverage is 1.03 times for male than female in urban area holding the other variables constant. The sixth or above child's expected vaccination coverage is .91 times less than the first child in both areas. Compared to Dhaka, vaccination rate is higher for Khulna and Rajshahi and lower for Sylhet and Chittagong in rural area. In urban area only Chittagong came as a division with significantly lower coverage than Dhaka. Expected immunization coverage increases with mother's education and rich economic status compared to non-educated mothers' and poor family. Again the expected frequency is greater for mothers' who read newspaper almost every day than those who do not read newspaper at all. Watching TV at least once a week also contributes significantly to the improvement of vaccination coverage in urban area than not watching at all. The significant constants indicate that, evaluating all the other charactering having no effect, it is expected that a child will be given approximately 8 of these 11 doses of vaccines. Alpha is the dispersion parameter. If the dispersion parameter equals zero, the model reduces to the simpler Poisson model. If the dispersion parameter, is significantly greater than zero than the data are over dispersed and are better estimated using a negative binomial model than a poison model. So, fitting Negative Binomial Regression model to the rural area and Poisson Regression model to the urban area is also correct in terms of the obtained Alpha. 


\section{Conclusion}

The paper investigates the determinants of vaccination coverage and how it differs between rural and urban area in Bangladesh. The results suggest that for both areas, children with birth order $6+$ is less vaccinated than first child. Compared to Dhaka, Khulna and Rajshahi are more and Sylhet and Chittagong are less vaccinated in urban area. Mother's education, reading newspaper almost every day and rich economic status is positively associated with immunization coverage for both areas where mothers' who watch TV at least once a week also increases the coverage in the urban area. The findings might assist policy-makers to recognize and minimize the inequalities in the vaccination coverage to improve the immunization status of under five children in Bangladesh.
1. Cameron, A.C. and P.K. Trivedi, 1998. Regression analysis of count data, Cambridge University Press.

2. Chowdhury A. M. R., A. Bhuiya, S. Mahmud, A. K. M. A. Salam, and F. Karim, 2003. Immunization Divide: Who Do Get Vaccinated in Bangladesh?, International Centre for Diarrhoeal Disease Research.

3. Hilbe, J.M., 2007. Negative Binomial Regression, Cambridge University Press.

4. NIPORT, Mitra and Associates and ORC Macro, 2007; Bangladesh demographic and health survey 2007.National Institute of Population Research and Training (NIPORT), Mitra and Associates and ORC Macro.

5. Quaiyum A., C. Tunon, H. A. Baqui and J. Khatun, 1997. Impact of National Immunization Days on Polio-related Knowledge and Practice of Urban Women in Bangladesh, International Centre for Diarrhoeal Disease Research. 